
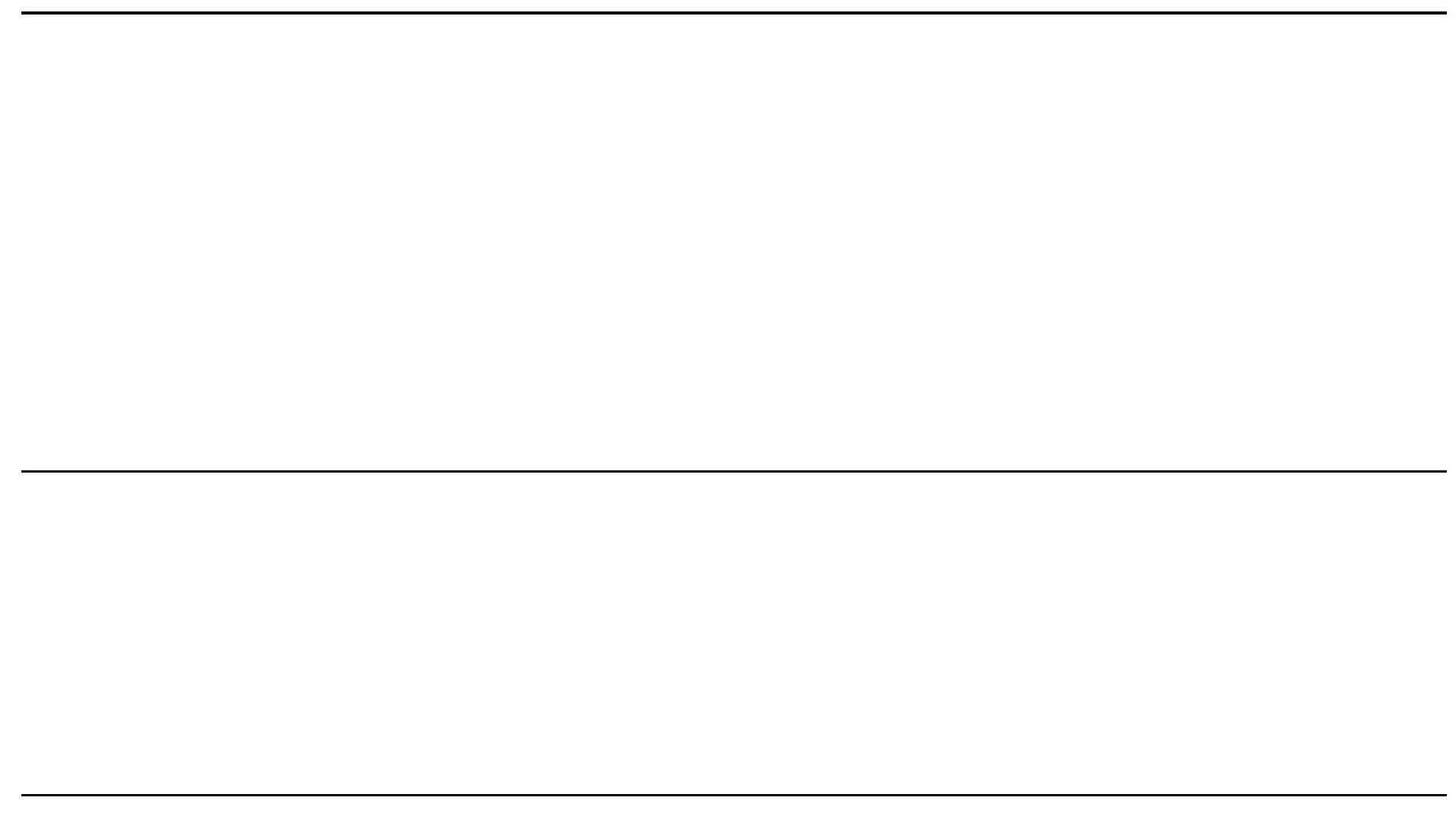


\section{ORIGINALARTICLE CLINICAL ONCOLOGY}

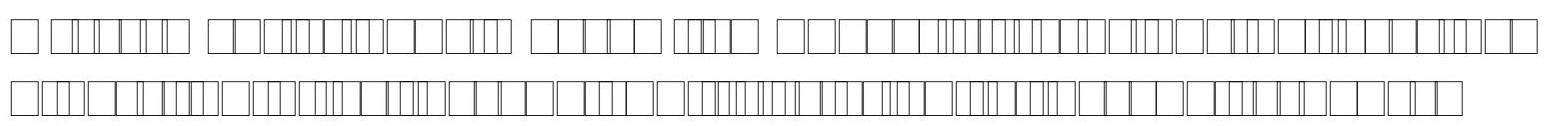

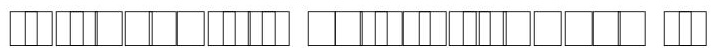

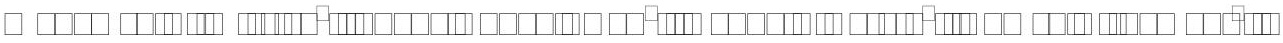

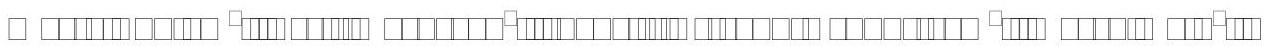

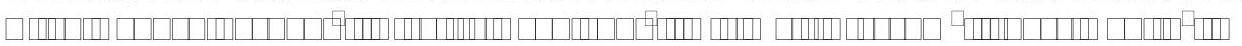

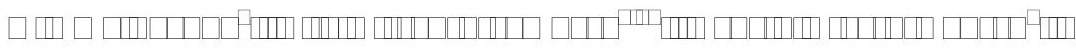

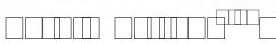

Received: 1 December 2015 / Accepted: 16 December 2015 / Published online: 8 J anuary 2016

' Springer-Verlag Berlin Heidel berg 2016

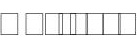

Purpose Tyrosine kinase inhibitors (TKIS) used in the treatment of chronic myeloid leukaemia have been reported to induce immunomodulatory effects. We aimed to assess peripheral blood (PB) and bone marrow (BM) lymphocyte status at the diagnosis and during different TKI therapies and correlate it with treatment responses.

Methods BM and PB samples were acquired from 105 rst-line TK I-treated patients. Relative number of BM Iymphocytes was evaluated from MGG-stained BM aspirates, and immunophenotypic analyses were performed with multicolour ow cytometry.

Results Early 3-month expansion of BM lymphocytes was found during all different TK Is (imatinib n 71, 20\%; dasatinib n 25, $21 \%$; nilotinib $n \quad$ 9, $22 \%$; heal thy controls $n \quad 14,12 \%, p<0.0001$ ). Increased PB lymphocyte count was only observed during dasatinib therapy. The BM lymphocyte expansion was associated with early molecular response; patients with 3-month $B C R$ ABL1

* Satu M ustjoki

satu.mustjoki@ helsinki.

1 Hematology Research U nit Helsinki, D epartment of Hematology, U niversity of Helsinki and Helsinki University Central Hospital Comprehensive Cancer Center, Haartmaninkatu 8, P.O. B ox 700, $00290 \mathrm{Helsinki,} \mathrm{Finland}$

2 Institute of Clinical M edicine, U niversity of Helsinki, Helsinki, Finland

3 Sk ne U niversity Hospital, L und, Sweden

4 Department of Hematology, U me University Hospital, Ume, Sweden

5 Department of Hematology, St Olavs Hospital, N orwegian University of Science and Technology (NTNU), Trondheim, Norway
$<10 \%$ showed higher lymphocyte counts than patients with BCR ABL $1>10 \%$ ( 23 vs. $17 \%, p<0.05$ ). Detailed phenotypic analysis showed that BM lymphocyte expansion consisted of various Iymphocyte subclasses, but especially the proportion of CD19 B cells and CD3negCD 16/56 NK cells increased from diagnostic values. During dasatinib treatment, the lymphocyte balance in both BM and PB was shifted more to cytotoxic direction (increased CD8 CD57 and CD8 HLA-DR cells, and low T regulatory cells), whereas no major immunophenotypic differences were observed between imatinib and nilotinib patients.

Conclusions Early BM lymphocytosis occurs with all current rst-line TKIs and is associated with better treatment responses. $\mathrm{PB}$ and $\mathrm{BM}$ immunopro le during dasatinib treatment markedly differs from both imatinib- and nilotinib-treated patients.

\section{B one marrow $\cdot$ Lymphocyte $\cdot \mathrm{CM} \mathrm{L} \cdot$ Tyrosine} kinase inhibitor $\cdot$ Therapy response

6 Department of Pathology, Rigshospitalet, University of Copenhagen, Copenhagen, Denmark

7 Department of Hematology, Rigshospitalet, University Hospital of Copenhagen, Copenhagen, Denmark

8 Department of Pathology, U ppsala U niversity H ospital, Uppsala, Sweden

9 Department of H ematology, U ppsala U niversity Hospital, Uppsala, Sweden

10 Department of Clinical Chemistry, University of Helsinki, Helsinki, Finland 






